\title{
The Inconsistency of Expected Utility Theory with Certain Classes of Single-Peaked Preference Functions ${ }^{1}$
}

\author{
Alexander Pollatsek ${ }^{2}$ \\ University of Michigan, Ann Arbor, Michigan 48104
}

Received June 23, 1969

\begin{abstract}
The "psychophysics of gambling" model, in which a person is assumed to have a single-peaked or monotonic preference function on each of the primary psychological dimensions of a gamble, is compared to the expected utility model for two-outcome gambles. Two theorems are proved, the first of which shows that expected utility (EU) theory is inconsistent with single-peaked preference functions on both skewness and variance, while the second shows that subjectively-expected utility (SEU) thcory is inconsistent with single-pcaked preferencc functions on both skewness and range. The applicability of the theorems is discussed and a weak test of SEU theory is made employing the second theorem.
\end{abstract}

\section{INTRODUCTION}

In decision making under risk, expectation theories have held the center of the stage for over a century. The first major expectation theory, historically, is expected value (EV) theory, which postulates that an individual selects the option with the highest expected monetary value. When EV theory was shown to have serious difficulties (e.g., the St. Petersburg paradox), another theory called expected utility (EU) theory was proposed (Bernoulli, 1738). In EU theory, every individual is postulated to have a utility function which is restricted only in that it must be continuous and it must be a monotonically increasing function of monetary value. EU theory postulates that the subject maximizes his expectation of utility. When EU theory was found to be a somewhat inadequate descriptive model, a subjective probability function was postulated (Edwards, 1955). In many discussions of subjective probability this function is restricted to be a continuous and monotonically increasing function of objective probability (Preston and Baratta, 1948; Coombs, Bezembinder and Goode, 1967). Subjectively-expected utility (SEU) theory predicts that the subject will maximize $\sum \psi\left(p_{i}\right) U\left(a_{i}\right)$ where $\psi\left(p_{i}\right)$ is the subjective probability of event $i$ having objective probability $p_{i}$ and $U\left(a_{i}\right)$ is the utility of the consequence $a_{i}$, of event $i$ (Edwards, 1955).

1 This work was supported by U.S.P.H.S. Grant MH-04236 while the author was a N.A.S.A. trainee.

${ }^{2}$ Now at Univ. of Massachusetts, Amherst, Mass. 01002. 
An alternative approach to the general class of expectation theories has been proposed by Coombs and Pruitt (1960) for two-outcome gambles. A two-outcome gamble is considered as a three-dimensional stimulus; the three dimensions selected by Coombs and Pruitt were expectation $(E)$, variance $\left(\sigma^{2}\right)$, and skewness $(S)$. For two outcomes with consequences $a$ and $b,(a>b)$ and with probabilities $p$ and $q$, respectively, these three quantities are given by

$$
\begin{aligned}
E & =p a+q b, \\
\sigma^{2} & =p q(a-b)^{2} \\
S & =\frac{1-2 p}{\sqrt{p q}} .
\end{aligned}
$$

The dimension of variance measures the spread of the bet. The dimension of skewness is monotonic with the probability of losing, and, therefore, skewness preference can also be viewed as probability preference or "odds" preference. To minimize confusion, the third dimension will be subsequently referred to as skewness.

Coombs and Pruitt concentrated their attention on the situation in which $\sigma^{2}$ or $S$ is varied with the other two dimensions held constant; since, if $\sigma^{2}$ and $S$ are the same for two two-outcome gambles, the gamble with the higher expectation will dominate the other. They predict that the subject will "fold" a series of bets varying only in skewness or in variance. The folding model postulates that variance and skewness are psychological dimensions and that the subject has an ideal point, or most preferred value, on each of these dimensions, and that the subject folds the dimension (line) at this ideal point. The subject's preference ordering of stimuli is then the ordering of the stimuli on the folded dimension. Luce and Raiffa (1957) have shown that this model predicts the same preference orderings as a model in which the subject has a single-peaked preference function on the relevant dimension. Coombs and Pruitt found that most of their subjects did have preference functions (rank orders) on both variance and skewness that conformed to the folding model, although the ideal points for skewness changed as the variance changed, and vice versa.

The folding model of two-outcome gambles is more vulnerable to a simple critical test than is SEU theory (compare the analysis of the Coombs and Pruitt (1960) experiment with that of the Coombs, Bezembinder and Goode (1967) experiment), and might be equivalent to a special case of SEU theory with well-defined restrictions on the utility and subjective probability functions. However, the exact correspondence between the two theories is largely an unsolved question. Given a specific utility function and a specific subjective probability function, it would be a simple matter of calculation to determine the subject's skewness and variance preferences, but there is little that can be said at present about his skewness and variance preferences if all that is known is that he obeys SEU theory. Discovery of the opposite type of correspondence seems 
to be an easier problem to solve; i.e., given a certain set of skewness preferences and variance preferences, what can one say about the subject's utility function and subjective probability function? A general solution to this second problem is also unsolved, but in certain specific cases the correspondence is known. For example, if a subject's preference for variance is monotonically increasing for all values of $E$ and $S$, then he will have a positively accelerating (concave upward) utility function. Similarly, if his preference for variance is monotonically decreasing, he will have a negatively accelerating (concave downward) utility function. On the other hand, if a subject has a single-peaked variance preference function with ideal variance not equal either to zero or infinity, then it can be shown that his utility function must. have at least one inflection point.

A more general question might be asked: Are all patterns of variance preference and skewness preference compatible with SEU theory? It would seem that all reasonable sets of folding patterns would be. An example of an "unreasonable" set of preferences would be this: A subject strongly preferred gamble $A$ to $B$, but reversed the order of preference if a penny were added to each outcome of both gambles. However, it can be shown that very reasonable sets of folding patterns do violate EU theory. The following theorem specifies such sets of folding patterns.

THEOREM 1. If the following conditions are true for two-outcome gambles, then the subject must violate expected utility theory:

1) The subject has a single-peaked preference function on stimuli (gambles) that vary in skewness with variance and expectation held constant, for all values of the parameters, expectation and variance, and with ideal skewness finite and bounded from above.

2) The subject has a preference pattern that is not monotonically increasing with variance if expectation and skewness are held constant, for any values of the parameters, expectation and skewness.

If the psychological space is reparameterized so that the dimensions varied are expectation, skewness, and range $(R=a-b)$, then the following theorem involving SEU theory can be stated.

Theorem 2. If the following conditions are true for two-outcome gambles, then the subject must violate subjectively-expected utility theory:

1') The subject has a single-peaked preference function on stimuli (gambles) that vary in skewness with range and expectation held constant, for all values of the parameters, expectation and range, and with ideal skewness taking any finite value.

$\left.2^{\prime}\right)$ The subject has a preference pattern that is not monotonically increasing with range if expectation and skewness are held constant, for any values of the parameters, expectation and skewness. 
A more informal statement of Theorem 2 is the following: If the subject always folds the skewness dimension somewhere other than at the end (note that the ideal point does not have to stay constant), then if he obeys SEU theory, he always prefers a higher range gamble to a lower range gamble having the same probabilities and expected values.

It should be emphasized that in assuming Conditions $I$ and 1 , one does not have to assume that the subject is ever asked to make a comparison between a two-outcome gamble and a sure thing; such comparisons are not assumed in the proofs that follow. The lemma below does assert that a consequence of Condition 1' is that any gamble has a higher utility than the utility of its cash equivalent (in terms of expected value). However, a direct test of this consequence is a bit tricky since, in some sense, gambles and sure things are different entities. A person may choose a sure thing because it is simpler than a gamble and not because it has a higher $S E U$ than the gamble. To repeat, the domain of these theorems is comparisons between pairs of nondegenerate (variance strictly greater than zero) two-outcome gambles.

It should be noted that since range is monotonic with variance for two-outcome gambles with fixed probabilities, range preference is indistinguishable from variance preference in a set of two-outcome gambles with fixed skewness. However, bets with constant range have different variances as skewness changes and bets of constant variance have different ranges as skewness changes $\left(\sigma^{2}=p(1-p) R^{2}\right)$.

Proof of Theorem 2. Theorem 2 will be proved first since it will be easier to see why the line of argument used does not lead to the proof of a more general theorem than Theorem 1. The theorem is proved by assuming Condition 1' and SEU theory and showing that condition $\left(2^{\prime}\right)$ cannot hold.

Lemma. For any set of gambles with expectation $E$ and range $R$, the subjectivelyexpected utility of the gamble is greater than the utility of $E$ if Condition $1^{\prime}$ and $S E U$ theory are true.

Any two-outcome gamble may be presented as a probability $p$ of obtaining $E+(1-p) R$ and probability $(1-p)$ of obtaining $E-p R$, where $p$ is the probability of obtaining the larger amount. This is true since any three independent parameters can fully describe a two-valued random variable. Remember that $1-p$ is monotonically related to skewness so that if $E$ and $R$ are held constant, probability preferences are the same as the skewness preferences specified in Theorem 2 . Then for any gamble with expectation $E$ and range $R$,

$$
S E U(\text { gamble })=\psi(p) \cdot U[E+(1-p) R]+[1-\psi(p)] \cdot U(E-p R)
$$

where $\psi(p)$ is the subjective probability of obtaining $E+(1-p) R$ and $1-\psi(p)$ is the subjective probability of obtaining $E-p R$. 
We know by Condition $1^{\prime}$ that the $S E U$ of the set of gambles with fixed $E$ and $R$ has a maximum value at some finite skewness $(p \neq 0,1)$ and therefore some $\psi(p) \neq 0,1$. The condition that the function is single-peaked implies that the $S E U$ decreases as $p$ approaches 0 or 1 . The question to be asked is whether the $S E U$ function has a limit as $p$ approaches 0 or 1 , and if so, what that limit is. For expectation $E$ and range $R$,

$$
\lim _{p \rightarrow 0} S E U(\text { gamble })=\psi(p) \cdot U(E+R)+[1-\psi(p)] \cdot U(E)=U(E),
$$

since as $p \rightarrow 0, \psi(p) \rightarrow 0$ and $1-\psi(p) \rightarrow 1$. Similarly,

$$
\lim _{p \rightarrow 1} S E U(\text { gamble })=\psi(p) \cdot U(E)+[1-\psi(p)] \cdot U(E-R)=U(E) .
$$

Since the function is minimal at the ends and the limit at the ends is $U(E)$, the function must be greater than $U(E)$ everywhere.

This completes the proof of the lemma.

Now we wish to show that Condition 2 ' cannot occur; that is we wish to prove that for all $R^{\prime}>R$, and all $E$ and $p$, the subject must prefer a gamble with the parameters $E, R^{\prime}$ and $p$ to a gamble with parameters $E, R$ and $p$. That is,

$$
\begin{aligned}
& \psi(p) \cdot U[E+(1-p) R]+[1-\psi(p)] \cdot U(E-R p) \\
& \quad<\psi(p) \cdot U\left[E+(1-p) R^{\prime}\right]+[1-\psi(p)] \cdot U\left(E-R^{\prime} p\right) .
\end{aligned}
$$

We know from the lemma, that for any $p, E, R(p \neq 0,1)$,

$$
\psi(p) \cdot U[E+(1-p) R]+[1-\psi(p)] \cdot U(E-p R)>U(E) .
$$

Consider the gamble: probability $1 / n$ of obtaining $E_{0}+n(1-p) R$ and probability $(1-1 / n)$ of obtaining $E_{0}$, with $n$ any real number greater than one. The expectation of this gamble is

$$
\frac{1}{n}\left[E_{0}+n(1-p) R\right]+\left(1-\frac{1}{n}\right)\left[E_{0}\right]=E_{0}+(1-p) R .
$$

Therefore, by the lemma

$$
\psi\left(\frac{1}{n}\right) \cdot U\left[E_{0}+n(1-p) R\right]+\left[1-\psi\left(\frac{1}{n}\right)\right] \cdot U\left(E_{0}\right)>U\left[E_{0}+(1-p) R\right] .
$$

Similarly, consider the gamble: probability $1 / n$ of obtaining $E_{0} \cdots n p R$ and probability $(1-1 / n)$ of obtaining $E_{0}$. By a computation similar to the above, the expectation of this gamble is equal to $E_{0}-p R$, where the $E_{0}, R, p$, and $n$ are the same as the ones in the above gamble. Therefore, by the lemma

$$
\psi\left(\frac{1}{n}\right) \cdot U\left(E_{0}-n p R\right)+\left[1-\psi\left(\frac{1}{n}\right)\right] \cdot U\left(E_{0}\right)>U\left(E_{0}-p R\right) .
$$


If one multiplies inequality (1) by $\psi(p)$ and inequality (2) by $1-\psi(p)$ and adds the two resulting inequalities and makes another simple substitution in the inequality, one gets the following inequality:

$$
\begin{aligned}
\psi(p) \cdot U\left[E_{0}+n(1-p) R\right]+[1-\psi(p)] \cdot U\left(E_{0}-n p R\right) \\
\quad>\psi(p) \cdot U\left[E_{0}+(1-p) R\right]+[1-\psi(p)] \cdot U\left(E_{0}-p R\right) .
\end{aligned}
$$

This is true for any $E_{0}, R, p$, and any $n>1$, which is what we set out to prove. Inequality (3) implies that for fixed probability of winning (therefore, fixed skewness) and fixed expectation, the subject will always prefer the bet with the bigger range, since the quantity on the left represents a gamble with expectation $E_{0}$, range $n R>R$, and probability of winning $p$; while the quantity on the right is the $S E U$ of a gamble with expectation $E_{0}$, range $R$, and probability of winning $p$.

Therefore, Condition $2^{\prime}$ is violated and Theorem 2 is proved.

Proof of Theorem 1. Following the proof of Theorem 2, Condition 1 and EU theory will be assumed and a contradiction will be found to Condition 2. To see why the argument analogous to the one in the previous proof does not violate Condition 2 if SEU theory is assumed, we will begin by assuming SEU theory and later restrict the assumption to EU theory (i.e., set $\psi(p)=p$ ).

If $E$ is the expectation, $p$ is the probability of winning the more preferred outcome, and $\sigma$ is the standard deviation, then any two-outcome gamble may be represented by a probability $p$ of obtaining $E+\sigma \sqrt{(1-p) / p}$ and a probability $(1-p)$ of obtaining $E-\sigma \sqrt{p / 1-p}$. If $E$ and $\sigma$ are held constant, then probability preferences are the same as the skewness preferences specified in Theorem 1. The $S E U$ of a gamble is thus given by

$$
S E U(\text { gamble })=\psi(p) \cdot U\left(E+\sigma \sqrt{\frac{1-p}{p}}\right)+[1-\psi(p)] \cdot U\left(E-\sigma \sqrt{\frac{p}{1-p}}\right)
$$

If we examine the limiting values of $S E U$ as $p$ approaches 0 or 1 , we obtain

$$
\begin{aligned}
& \lim _{p \rightarrow 0} S E U(\text { gamble })=\psi(p) \cdot U\left(E+\sigma \sqrt{\frac{1-p}{p}}\right)+U(E) \geqslant U(E), \\
& \lim _{p \rightarrow 1} S E U(\text { gamble })=U(E)+[1-\psi(p)] \cdot U\left(E-\sigma \sqrt{\frac{p}{1-p}}\right)=?
\end{aligned}
$$

Since $S E U$ is assumed to be a single-peaked function of $p$, we know that its limit as $p \rightarrow 0$ is at least as big as $U(E)$, since the smallest limit the first term in (4) can have is zero. However, the limit of $S E U$ as $p \rightarrow 1$ can easily be smaller than $U(E)$, so that it is only certain that $S E U$ (gamble) will be greater than $U(E)$ for 
all probabilities that are less than or equal to the ideal probability. Thus, it is not clear exactly what restrictions on variance preferences can be deduced assuming SEU theory and Condition 1.

However, if we assume EU theory instead, then a contradiction to Condition 2 follows quite simply. Since we have assumed that the ideal probabilities are all greater than or equal to $p_{0}>0$ (this is equivalent to assuming that the ideal skewness is bounded from above), then it is true that the $E U$ of all gambles with probability $p_{\mathbf{0}}$ of winning the more desirable outcome are greater than the utility of the expectation of those gambles (by 4). It can easily be shown (Hardy, Littlewood, and Polya, 1934, p. 73) that for each $x_{1}$ and $x_{2}$ if there is some $p$ such that

$$
p \cdot U\left(x_{1}\right)+(1-p) \cdot U\left(x_{2}\right)>U\left[p x_{1}+(1-p) x_{2}\right],
$$

then $U$ is concave upwards. Since we know that this inequality is satisfied for any $x_{1}$ and $x_{2}$ by setting $p$ equal to $p_{0}, U$ must be concave upwards (positively accelerating). If $U$ is concave upwards, then it is a simple matter to show that the variance preferences are monotonically increasing and therefore Condition 2 must be violated.

\section{Discussion}

The above theorems describe quite general sets of preference patterns that violate EU theory and SEU theory, respectively. There are two major problems, however, in utilizing these theorems to test the appropriate utility theory.

The first problem is that it is hazardous to infer that all of a subject's skewness preferences are single-peaked from the observation that those skewness preferences sampled by the experiment were all single-peaked. While it is probably reasonable to interpolate and assume that behavior found for gambles at two different expectations that are not too far apart will also be found for gambles at all intermediate values of expectation, it is probably unreasonable to extrapolate in the same way. That is, it would be desirable to obtain versions of the two theorems that would apply to a region of values so that one isn't forced to extrapolate and assume that the subject would behave the same way if he were playing for hundreds of dollars as he does in the laboratory.

For Thcorem 2, a "restricted" version can be stated quite simply:

THEOREM 2a. If a subject obeys SEU theory and has single-peaked (ideal is finite) skewness preferences for two-outcome gambles for all $E$ such that $x_{1} \geqslant E \geqslant x_{2}$ and for all $R$ such that $R \leqslant x_{1}-x_{2}$, then for any two two-outcome gambles with equal $E$ and $p$ whose outcomes are between $x_{1}$ and $x_{2}$, the gamble with the greater range must be preferred. 
A truly "restricted" version of Theorem 1 would be rather difficult to state since a single skewness preference function for fixed $E$ and $\sigma^{2}$ involves indefinitely large positive outcomes and indefinitely large negative outcomes (see 4 and 5). However, the following "restricted" theorem is of some interest.

Theorem la. If the subject obeys $\mathrm{EU}$ theory and has single-peaked (ideal is finite and less than some maximum) skewness preferences for two-outcome gambles for all E such that $x_{1} \geqslant E \geqslant x_{2}$ and for all $\sigma$ such that $\sigma \leqslant \frac{1}{2}(a-b)$, then for any two gambles with equal $E$ and $p$ whose outcomes are between $x_{1}$ and $x_{2}$, the gamble with the greater variance must be preferred.

Thus, if one is willing to assume that one can interpolate between the expectations and variances or ranges at which skewness preferences are tested, then Theorems Ia and $2 \mathrm{a}$ can be used to test expectation theories.

In addition to the interpolation-extrapolation problem there is the problem that the class of situations in which subjects give single-peaked skewness preferences has not been clearly established. Coombs and Pruitt (1960) found that about $60 \%$ of their subjects gave single-peaked skewness preferences (ideal skewness not necessarily finite). On the other hand, Slovic, Lichtenstein, and Edwards (1965) and Pollatsek (1966) obtained lower proportions of subjects who gave single-peaked skewness preferences: $11 \%$ and $30 \%$, respectively. In Pollatsek's experiment, range was held constant and in the other two, variance was held constant.

Slovic, Lichtenstein, and Edwards (1965) hypothesized that the proportion of singlc-pcaked skewness preferences in Coombs and Pruitt's experiment was high only because the subjects were bored and that a less boring (i.e., more realistic) gambling situation would lead to more complex preferences. Accordingly, they attempted to manipulate boredom and found some confirmation for their hypothesis. Their "boring" condition approximated Coombs and Pruitt's experimental situation in that the subjects were run in groups and the gambles were not actually played, while their "motivated" condition employed frequent playing of the gambles and individual running of the subjects. This manipulation is likely to be changing many variables other than boredom (e.g., complexity of the perceived task). Hence, the presence of more single-peaked skewness preferences in the Slovic, et al., "boring", condition than in their "motivated" condition can only be considered tentative evidence for their "boredom" hypothesis.

In both the Coombs-Pruitt and Slovic, et al., experiments, preferences were tested only at $E=0$ so that even Theorem 1 a is not really appropriate for testing EU theory. However, Pollatsek's (1966) data allow for a test of SEU theory since each subject was tested for his skewness preference at three values of expectation $(-60 \notin, 0 \phi, 60 \phi)$ and at 3 different values of range $(20 \phi, 40 \phi, 60 \phi)$. If all of a subject's skewness preferences were single-peaked (finite ideal), they were considered to satisfy Condition 
1' of 'Theorem 2. Of the four subjects (out of 67) who satisfied Condition 1', only two had range preferences that were all monotonically increasing. 'T'here were nine other subjects who were close to satisfying Condition $1^{\prime}$ and none of them were close (see the following paragraph) to having every range preference monotonically increasing as SEU theory combined with Condition $1^{\prime}$ would predict.

In using Theorem 2a to test SEU theory one can predict only that the subject should have monotonically increasing preference for range for gambles with expected value equal to zero (assuming SEU theory and Condition 1'). Each of the 11 subjects who violated SEU theory by the previous test had a range preference function at zero expected value that was essentially monotonically decreasing and therefore seriously in violation of SEU theory using Theorem 2a. Of course, the fact that only 13 out of the 67 subjects gave only single-peaked preference functions is no evidence that the other 54 subjects were obeying SEU theory.

The above application of 'Theorems 2 and 2a to Pollatsek's data illustrates how the theorems of this paper can be utilized to provide experimental tests of EU or SEU theory. However, the test using the above data is somewhat weak. On the positive side, Theorem $2 \mathrm{a}$ assumes only interpolation with the skewness preferences, which seems quite reasonable. Furthermore, the rejection of the predicted monotonically increasing range preference functions for 11 of the 13 subjects is unequivocal. The major weakness of the above test is that only four out of 67 subjects gave perfect skewness folding patterns and that the other 9 subjects are included as skewness folders even though their patterns are not perfect. Since 54 subjects did not appear to be folding skewness (63 subjects by a strict criterion), it is not unreasonable that the skewness folding patterns that obtained in the above experiment were artifacts of some other decision process. It follows, therefore, that the place of the psychophysics of gambling framework and its implications for expectation theories will be clear only when the experimental conditions that produce reliable skewness preferences are fully understood.

\section{REFERENCES}

Bernovlli, D. Specimen theoriae novae de mensura sortis. Comentarii academaie scientiarium imperiales petropolitanae, 1738, 5, 175-192. (Trans. by L. Sommer in Econometrica, 1954, 22, 23-36.)

Coombs, C. H., and PruitT, D. G. Components of risk in decision making: Probability and variance preferences. Journal of Experimental Psychology, 1960, 60, 265-277.

Coombs, C. H., Bezembinder, T. G. G., ANd Goode, F. M. Testing expectation theories of decision making without measuring utility or subjective probability. Journal of Mathematical Psychology, 1967, 4, 72-103.

EDwards, W. The prediction of decisions among bets. Journal of Experimental Psychology, 1955, 50, 201-214.

Hardy, G. H., Littlewood, J. E., And Polya, G. Inequalities. Cambridge, England: The University Press, 1934. 
Luce, R. D., And Raiffa, H. Games and Decisions: Introduction and Critical Survey. New York: Wiley, 1957.

Pollatser, A. On the relation between subjectively expected utility theory and the psychophysics of gambling. Michigan Mathematical Psychology Program, Technical Report, 1966, MMPP 66-1. Univ. of Michigan, Ann Arbor, Mich.

Preston, M. G., ANd Baratta, P. An experimental study of the auction-value of an uncertain outcome. American Journal of Psychology, 1948, 61, 183-193.

Slovic, P., Lichtenstein, S., And Edwards, W. Boredom-induced changes in preferences among bets. American Journal of Psychology, 1965, 78, 208-217. 